\title{
Influence of light on the magnetoelectric effect in Fe-doped $\mathrm{Bi}_{12} \mathrm{SiO}_{20}$
}

\author{
Krzysztof Filar and Victor I. Nizhankovskii ${ }^{a}$ \\ Institute of Low Temperature and Structure Research, Polish Academy of Sciences, ul. Okolna 2, 50-422, Wroclaw, \\ Poland
}

Received 5 May 2017 / Received in final form 29 September 2017

Published online 15 January 2018

(C) The Author(s) 2018. This article is published with open access at Springerlink.com

\begin{abstract}
We report an extraordinary oxidation state of iron in the Fe-doped $\mathrm{Bi}_{12} \mathrm{SiO}_{20}$ single crystal. Magnetoelectric effect and changes of this effect under illumination of the sample were investigated in magnetic fields up to $32 \mathrm{~T}$. Moreover, an absorption line and its shift to higher energy caused by magnetic field (the Zeeman effect) were seen in the optical measurements. Obtained results show that the iron dopant is in unexpected, +4 oxidation state and it changes to +5 after illumination with blue light.
\end{abstract}

\section{Introduction}

Influence of light on the magnetic properties is known for a long time: photo-induced magnetism of a Co-Fe cyanide was reported in [1], sensitivity of electronic paramagnetic resonance (EPR) to the illumination was established in many works since 1982 [2,3]. Recently, we have observed a change of sign of the magnetoelectric effect in Cr-doped $\mathrm{Bi}_{12} \mathrm{TiO}_{20}$ after blue-light exposure [4]. All these phenomena result from changes in the oxidation state of $3 \mathrm{~d}$ element used as a dopant.

In the present work we investigate the influence of light on the magnetoelectric effect in Fe-doped $\mathrm{Bi}_{12} \mathrm{SiO}_{20}$ single crystal. Sillenites, such as our crystal, are materials with non-centrosymmetric cubic structure with space group I23 [5,6]. As it was discussed earlier [4], the magnetoelectric effect in this noncentrosymmetric crystal induces charge $Q$ at (1 00 ) faces of the sample if the magnetic field $\mathbf{H}$ is applied along the [0 111$]$ axis. The induced charge is proportional to the square of the magnetic moment

$$
Q=\beta M^{2}
$$

If dopant concentration is small, then its magnetic moment may be described by the Brillouin function

$$
\begin{aligned}
B_{J} & =\frac{2 J+1}{2 J} \operatorname{coth}\left(\frac{2 J+1}{2 J} y\right)-\frac{1}{2 J} \operatorname{coth}\left(\frac{y}{2 J}\right), \\
y & =J g \mu_{B} H / k_{B} T
\end{aligned}
$$

where $J$ is the total moment, $g=2$ is the Landé $g$-factor, $\mu_{B}$ is the Bohr magnetron, $k_{B}$ is the Boltzmann constant.

\footnotetext{
${ }^{\mathrm{a}}$ e-mail: v.nizhan@gmail.com
}

Usually the orbital moment of $3 \mathrm{~d}$ ions is frozen, so the total magnetic moment $J$ can be replaced by the total spin $S$.

In our previous work we obtained unexpected chromium +2 oxidation state [4]. Based on that investigation, we have also expected unusual oxidation state of iron in Fedoped $\mathrm{Bi}_{12} \mathrm{SiO}_{20}$.

\section{Experiment}

Large Fe-doped $\mathrm{Bi}_{12} \mathrm{SiO}_{20}$ single crystal was grown at the Ioffe Institute of physics and technology (St Petersburg, Russian Federation). Samples with appropriate orientation and size were cut with a wire saw. The sample for the magnetoelectric measurements was a parallelepiped $4.4 \times$ $4 \times 3.5 \mathrm{~mm}^{3}$. Electrodes (silver paint) were deposed on (1 000$) 4.4 \times 4 \mathrm{~mm}^{2}$ faces. Faces $(0 \overline{1} 1) 4.4 \times 3.5 \mathrm{~mm}^{2}$ were used for illumination and polished for better light penetration. Magnetic field was applied along [0 11 ] direction (the longest $4.4 \mathrm{~mm}$ dimension).

Experimental techniques used for the magnetic and magnetoelectric measurements were described in [4]. Geometry of experiment in a long-pulse magnetic field is shown in Figure 1a. Magnetic field is aligned along [ $\left.\begin{array}{lll}0 & 1 & 1\end{array}\right]$ direction, charge is measured at (1 000$)$ faces. Blue $\left(\lambda_{\max }=470 \mathrm{~nm}\right)$ and red $\left(\lambda_{\max }=640 \mathrm{~nm}\right)$ SMD LEDs (Kingbright Electronic Co., Ltd.) are placed at opposite polished faces of the sample.

We have also investigated optical properties and the Zeeman effect in a stationary magnetic field up to $14 \mathrm{~T}$ and in a long-pulse field up to $30 \mathrm{~T}$. The optical insert (Fig. 2b) permits measurements of the light absorption along (slot 3 ) and perpendicular (slot 5) to the magnetic field direction. Quartz fibre (diameter $0.6 \mathrm{~mm}$ ) was used as a beamguide. Blue (ProLight 3W PM2B-3LBS-SD) 
a)

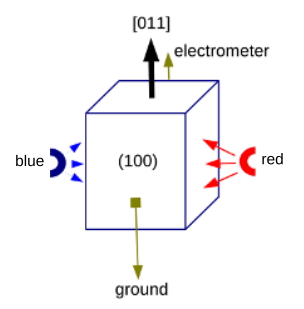

Fig. 1. Schematics of the experiments. (a) Geometry of sample mounting for the magnetoelectric measurements in long-pulse magnetic field. (b) Schematics of the optic cell. 1 - quartz fibres, 2 - lenses, 4 - reflection prisms, 3,5 -slots for a sample.

and white (HUEY JANN 5W HPE8b-49K5YWGA) power LEDs were used as sources of light emission. Spectra were analyzed with a polychromator (diffraction grating of 1800 lines $/ \mathrm{mm}$ ) and registered by a digital CCD line camera (CCD-S3600-D, ALPHALAS).

The sample for optical investigation was a polished (1 00 ) plate $0.49 \mathrm{~mm}$ thick. In the Faraday configuration (sample in slot 3) magnetic field and light were parallel to the $\left[\begin{array}{lll}1 & 0 & 0\end{array}\right]$ axis. In the Voigt configuration (sample in slot 5) light was directed along the $\left[\begin{array}{lll}1 & 0 & 0\end{array}\right]$ axis and magnetic field - along the [0 111$]$ axis.

\section{Results}

Figure 2 shows magnetic moment of Fe-doped $\mathrm{Bi}_{12} \mathrm{SiO}_{20}$ measured at $T=1.55$ and $4.25 \mathrm{~K}$. It consists of a diamagnetic contribution of the host crystal with a linear dependence on the magnetic field and a paramagnetic one of the dopant which saturates with the increasing field

$$
M=\chi H+m_{s} B_{J}(H, T) .
$$

We used a nonlinear least-square method for simultaneous fitting of the data obtained at two temperatures [7]. Assuming that $\chi$ is temperature-independent and putting $J=2$ we got $m_{s}=25.5 \mathrm{emu} / \mathrm{mol}$ and $\chi=$ $-5.31 \mathrm{emu} / \mathrm{T}$ mol. The mean square error of this fitting was $M S E=0.29$. Obtained value of $m_{s}$ corresponds to $0.11 \mathrm{~mol} \%$ of $\mathrm{Fe}^{4+}$. Visually fitting for $J=5 / 2\left(\mathrm{Fe}^{3+}\right)$ looks as well as for $J=2$, but resulting $M S E=0.38$ is $30 \%$ larger.

Results for the magnetoelectric effect measured in a long-pulse magnetic field at $T=4.2 \mathrm{~K}$ are presented in Figure 3. Before the illumination, induced electric charge is positive (curve 1). After 20 min of illumination with blue light the magnetoelectric effect changes the sign (curve 2). Subsequent $20 \mathrm{~min}$ of illumination with red light restore positive sign (curve 3 ), additional 20 min of illumination results in small increase of signal (curve 4). It should be noted that illumination of a virgin sample with red light did not change the magnetoelectric effect.

Without illumination, induced electric charge monotonically increases with field, whereas total magnetic moment goes through zero at $H \approx 4 \mathrm{~T}$. This means that diamagnetism of the host crystal does not contribute to the

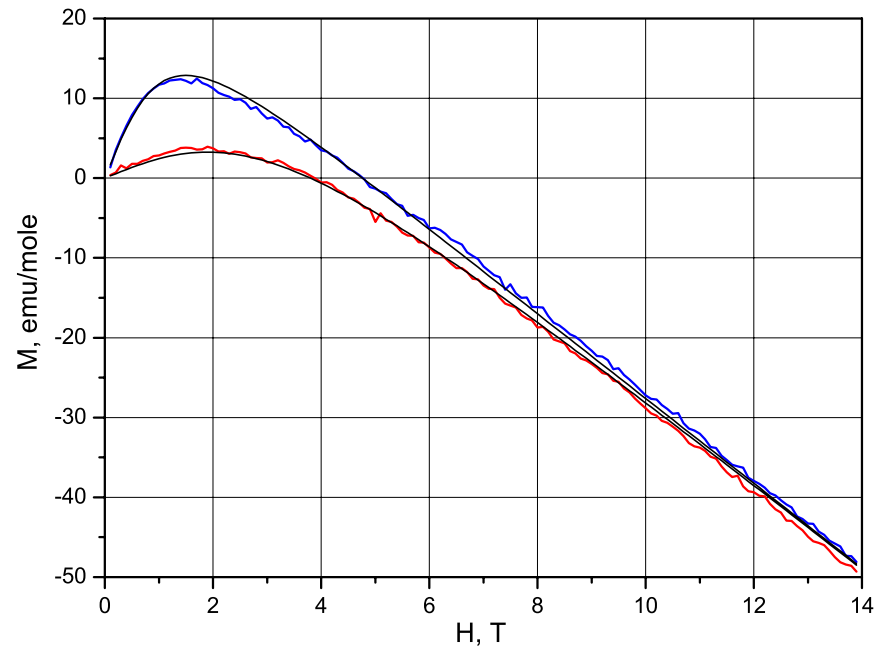

Fig. 2. Magnetic moment of Fe-doped $\mathrm{Bi}_{12} \mathrm{SiO}_{20}$ single crystal measured at $T=4.25 \mathrm{~K}$ (bottom curve) and $T=1.55 \mathrm{~K}$ (upper curve). Thin lines - simultaneous fitting to equation (4) for $J=2$.

magnetoelectric effect. On the other hand, induced electric charge continues to grow at highest magnetic fields, where paramagnetic contribution saturates. Thus, besides paramagnetic moment of iron dopant, the effective magnetic moment of equation (1) should contain a linear term that responds to the magnetization of dopant environment

$$
Q=\frac{a_{1}}{2}\left(B_{2}+a_{2} H\right)^{2},
$$

where $B_{2}$ is defined by equation (2).

After illumination with blue light the magnetoelectric effect changes the sign. This results from the photorefractive effect that changes the oxidation state of iron ions, so terms similar to equation (5), but with smaller $J$ and different coefficients $a_{i}$, should be added to the phenomenological description of the magnetoelectric effect in the light-exposed samples.

Figure 4 shows the derivative of the magnetoelectric effect measured in stationary magnetic fields using the field-modulation technique and lock-in signal detection. Before the illumination and after a short $1 \mathrm{~s}$ exposure the derivative has a maximum at $H \approx 2 \mathrm{~T}$ for $T=4.25 \mathrm{~K}$. This position corresponds to the value calculated for $J=$ 2 , see Table 1 in [4]. ${ }^{1}$ Dramatic change of the derivative is seen after illumination with the blue light. Not only was a change of value obtained but, as previously [4], also the change in sign of the effect.

Experimental records are well fitted to the derivative of series containing only two terms with $J=2$ and $3 / 2$

$$
Q=\frac{a_{1}}{2}\left(B_{2}+a_{2} H\right)^{2}+\frac{a_{3}}{2}\left(B_{3 / 2}+a_{4} H\right)^{2} .
$$

Fitting parameters are presented in Table 1.

\footnotetext{
${ }^{1}$ In caption to this Table $1 d B_{2}^{2} / d H$ should be replaced by $d B_{J}^{2} / d H$.
} 


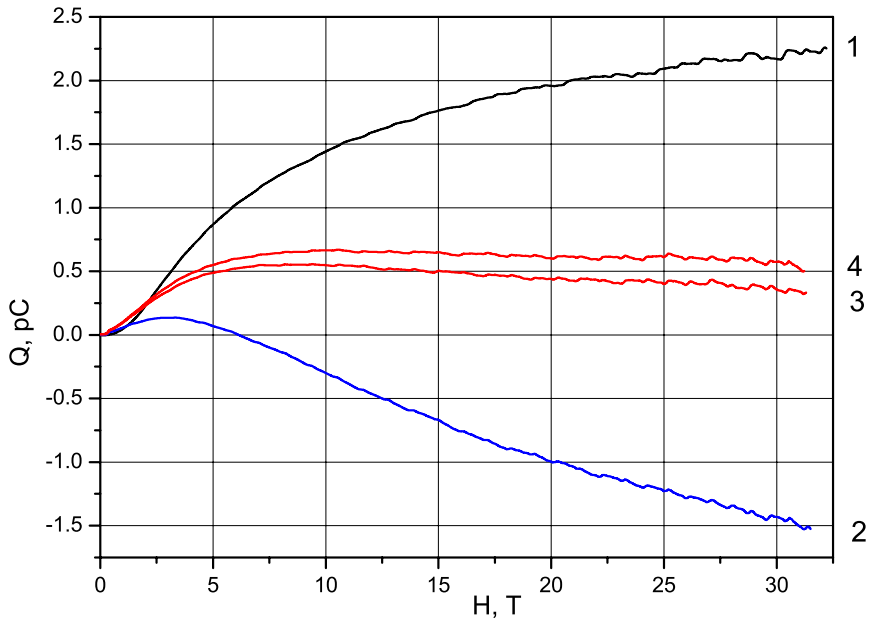

Fig. 3. Magnetoelectric effect of Fe-doped $\mathrm{Bi}_{12} \mathrm{SiO}_{20}$ single crystal measured in long-pulse magnetic field at $T=4.2 \mathrm{~K}$. Curve 1 - before the illumination, curve 2 - after 20 min exposure to blue light, curves 3 and 4 - after subsequent 20 and $40 \mathrm{~min}$ exposure to red light, respectively.

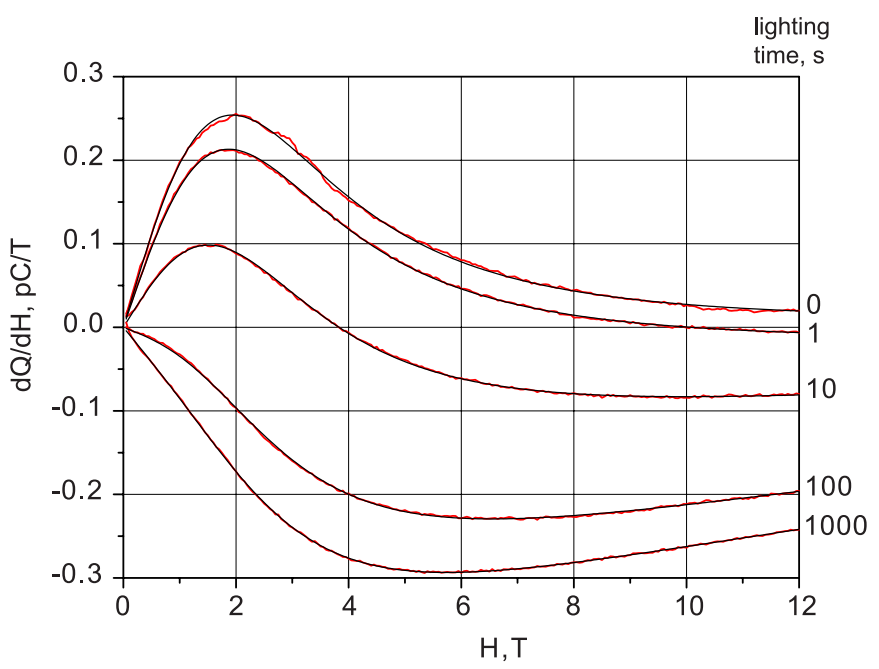

Fig. 4. Influence of the blue-light exposure on derivative of the magnetoelectric charge measured at $T=4.25 \mathrm{~K}$. Thin lines are fitting the curves by the derivative of equation (6) with parameters given in Table 1.

Influence of red-light illumination on the magnetoelectric effect in virgin Fe-doped $\mathrm{Bi}_{12} \mathrm{SiO}_{20}$ was almost negligible. After a very long (about $3 \mathrm{~h}$ ) exposure maximal signal $d Q / d H$ at $H=2 \mathrm{~T}$ was only $6 \%$ smaller. But if the sample was exposed to the blue light and as a result the magnetoelectric effect has an opposite sign, then illumination with the red light restore the initial sign of the effect.

In explaining the influence of illumination on the magnetoelectric effect in Cr-doped $\mathrm{Bi}_{12} \mathrm{TiO}_{20}$ we paid attention to the different transformation properties of the electron wave function with integer and half-integer total spin [4] that explain opposite sign of their contributions to the magnetoelectric effect. The same is valid for Fe-doped $\mathrm{Bi}_{12} \mathrm{SiO}_{20}$. Under illumination, the number of
Table 1. Results of the non-linear fitting of the experimental records $d Q / d H$ to the derivative of equation (6).

\begin{tabular}{lllll}
\hline $\begin{array}{l}\text { Lighting time } \\
(\mathrm{s})\end{array}$ & $\begin{array}{l}a_{1} \\
(\mathrm{pC})\end{array}$ & $\begin{array}{l}a_{2} \\
(1 / \mathrm{T})\end{array}$ & $\begin{array}{l}a_{3} \\
(\mathrm{pC})\end{array}$ & $\begin{array}{l}a_{4} \\
(1 / \mathrm{T})\end{array}$ \\
\hline 0 & 2.24 & 0.005 & - & - \\
1 & 2.08 & -0.007 & - & - \\
10 & 1.82 & -0.062 & -0.016 & 0.49 \\
100 & 1.45 & -0.17 & -0.055 & 0.77 \\
1000 & 1.24 & -0.22 & -0.089 & 0.76 \\
\hline
\end{tabular}

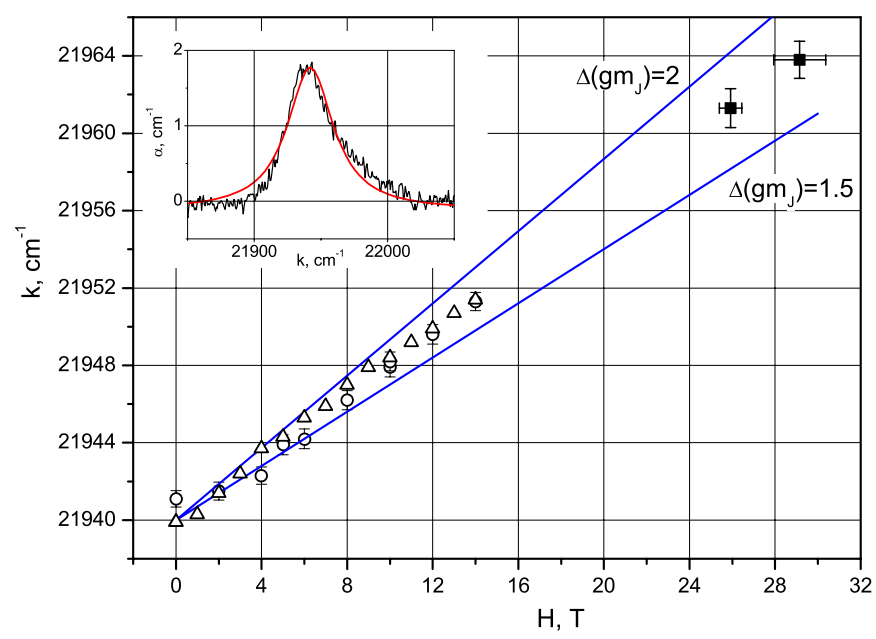

Fig. 5. Influence of magnetic field on position of the absorption line. Open circles and filled squares $-\mathbf{H} \|\left[\begin{array}{lll}1 & 0 & 0\end{array}\right]$, triangles $\mathbf{H} \|\left[\begin{array}{lll}0 & 1 & 1\end{array}\right]$. Insert: line recorded at $H=0$ and its Lorentzian fit.

$\mathrm{Fe}^{4+}$ ions gradually reduces and $\mathrm{Fe}^{5+}$ increases due to the photorefractive effect that is clearly seen from Table 1.

In optical investigations performed at $T=4.2 \mathrm{~K}$, we have observed a line near the absorption edge (at room and liquid nitrogen temperatures the line was invisible). This line after removing large background is shown in the insert of Figure 5. Width of the line is rather large $\left(w=40 \pm 2 \mathrm{~cm}^{-1}\right)$ due to a spin-orbit interaction [8]. Broadening of line is asymmetric, which can be explained by the non-centrosymmetric position of iron in the lattice. Magnetic field shifts the line to higher energy (the Zeeman effect). The effect was independent of mutual orientation of magnetic field, light beam and direction of magnetic field respective to the crystal axes.

The Zeeman effect was observed with the white LED as the light source, and the amplitude of the absorption line did not change during the experiment. When the white LED was replaced by the blue one, clear dependencies of absorption on the duration of illumination were obtained.

For this purpose blue LED with $\lambda_{\max }=454 \mathrm{~nm}$ was used (this wavelength coincides with the position of $\mathrm{Fe}^{4+}$ absorption line). After switching-on illumination, the transmission spectrum was registered several times with increasing time intervals. Each registration lasted one second. Absorption coefficient $\alpha$ was calculated according to Burger's law $\alpha=\ln \left(I_{0} / I\right) / d$ were $I_{0}$ is the incoming intensity, $I$ - registered intensity, $d$ - thickness of the sample. 


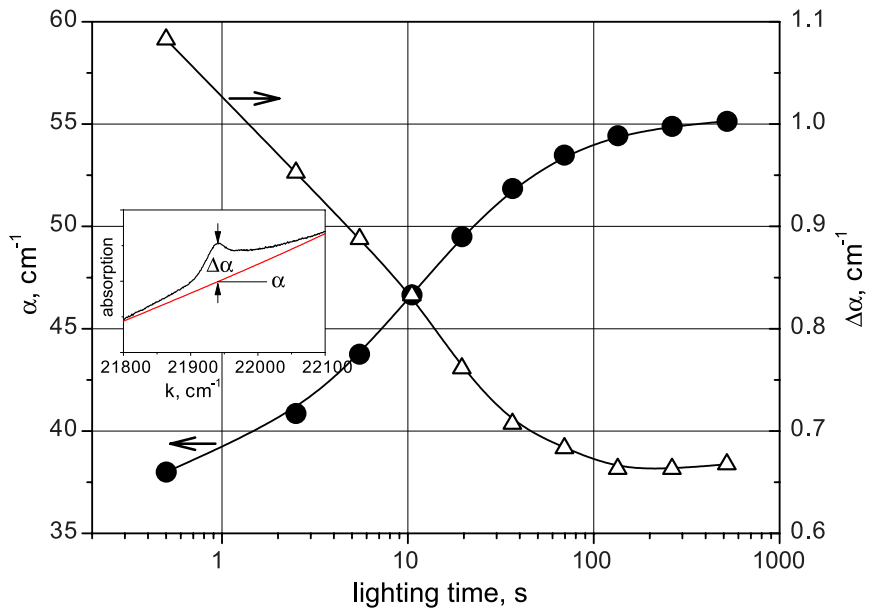

Fig. 6. Results of optical measurements with the blue light illumination at $T=4.2 \mathrm{~K}$. Insert shows definitions of the background absorption $(\alpha)$ and absorption caused by $\mathrm{Fe}^{4+}$ ions $(\Delta \alpha)$.

Experimental results are presented in Figure 6. Insert shows dependence of absorption on the wave number $k$. Absorption line of $\mathrm{Fe}^{4+}$ dopant looks as a bell curve on large background which increases with $k$. The background absorption gradually increases from $37 \mathrm{~cm}^{-1}$ at the beginning of illumination and saturates to $55 \mathrm{~cm}^{-1}$ with increasing time above $100 \mathrm{~s}$. Absorption of $\mathrm{Fe}^{4+}$ dopant diminishes from $1.1 \mathrm{~cm}^{-1}$ at the beginning to $0.66 \mathrm{~cm}^{-1}$ after $100 \mathrm{~s}$ of illumination. Thus the number of $\mathrm{Fe}^{4+}$ ions diminishes with the blue light illumination, that is in agreement with the results of the magnetoelectric effect measurements.

\section{Discussion}

The iron dopant is assumed to reside at the tetrahedral $\mathrm{Si}$ site. If doping with Fe retains compound stoichiometry, then it is logical to assume that both Fe and Si cations have $4+$ oxidation states. Our results for magnetization and magnetoelectric effect confirm this assumption. One more strong confirmation follows from optical investigation.

The $3 \mathrm{~d}$ shell of ion inserted in tetrahedral environment is split into two levels - the lower doublet ${ }^{5} \mathrm{E}$ and upper triplet ${ }^{5} \mathrm{~T}_{2}$ [9]. Orbital degeneracy is determined by the number of ways in which the electrons may be distributed on orbitals with the same energy. In the case of four electrons, each level is occupied by two electrons with the same spin direction (the first Hund rule). Both orbitals of the level ${ }^{5} \mathrm{E}$ are occupied, so it is an orbital singlet with $L=0, S=1, J=1, g=2$. Level ${ }^{5} \mathrm{~T}_{2}$ has two occupied and one unoccupied orbital, thus it is an orbital triplet with $L=1, S=1, J=2$ (the second Hund rule), $g=1.5$. The Zeeman effect in magnetic field results in an additional splitting (see Fig. 7). Two possible optical transitions are shown by vertical arrows. Initial states are occupied level 1 with $m_{J}=-1$ and level 2 with $m_{J}=0$. For final states one has three empty levels 6-8. Transition

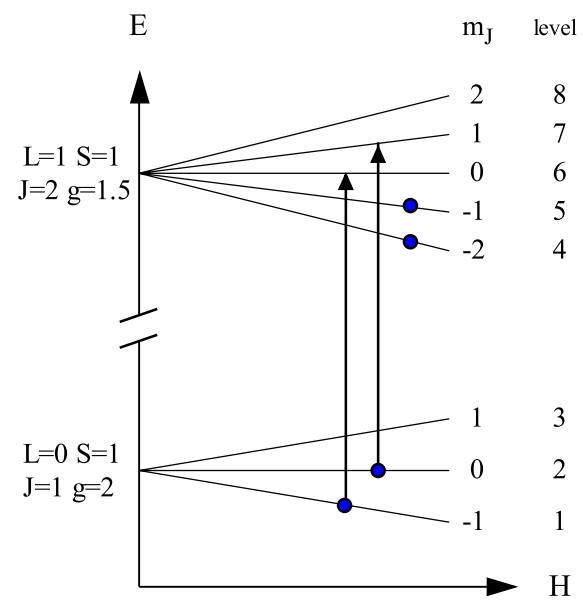

Fig. 7. Schematics of $3 \mathrm{~d}^{4}$ ion levels at tetrahedral position in magnetic field. Vertical arrows show optical transitions observed in the work.

from level 2 to level 6 is forbidden because in the initial and final states $m_{J}=0$. Transition to level 8 is forbidden because $\Delta m_{J}=2$. Thus only transition $2 \rightarrow 7$ is allowed. For initial state at level 1 transition to final state at level 6 with $m_{J}=0$ is allowed. Magnetic field will shift these transitions by

$$
\Delta k=\frac{\mu_{B} H}{h c}\left[\left(g m_{J}\right)^{f}-\left(g m_{J}\right)^{i}\right]
$$

where indexes $f$ and $i$ denote final and initial states respectively. Numerical value of the coefficient $\mu_{B} / h c$ is $0.467 \mathrm{~cm}^{-1} / \mathrm{T}$. For transition $1 \rightarrow 6 \Delta\left(g m_{J}\right)=2$, for transition $2 \rightarrow 7 \Delta\left(\mathrm{gm}_{J}\right)=1.5$. Corresponding lines are shown in Figure 5 . At field of $30 \mathrm{~T}$ the distance between transitions should be of about $\Delta k \approx 7 \mathrm{~cm}^{-1}$ that is 6 times smaller than width of the observed line. Figure 8 shows modelling of the absorption line. At $H=0$ both transitions coincide and observed absorption line has doubled amplitude of each transition. At $H=30 \mathrm{~T}$ transitions shift to higher energy by different amount, but resulting absorption line does not split. Line has Lorentzian shape with slightly smaller amplitude. Its width is approximated by $w(\Delta k) \approx w(0)\left[1+1.1(\Delta k / w(0))^{2}\right]$ and changes from $40 \mathrm{~cm}^{-1}$ at $H=0$ to $41.6 \mathrm{~cm}^{-1}$ at $H=30 \mathrm{~T}$, that is too small for our resolution. Consequently line splitting could not be resolved and an average of two predicted transitions with approximately equal intensities is observed in magnetic field. As it is seen from Figure 5, this simple qualitative consideration describes the experimental results very well.

The area under the absorption line $A=\int \alpha d k$ is proportional to the oscillator strength $f$ and to the dopant concentration $c=1.1 \times 10^{-3} \mathrm{~mol} \%$, which was determined from the paramagnetic contribution to the magnetization. From data shown in insert of Figure $5, A \approx 150 \mathrm{~cm}^{-2}$ that results in $f=4.32 \times 10^{-9} A / c \simeq 6 \times 10^{-4}$. This value is reasonable for the allowed electric-dipole transitions.

To observe the absorption, two rigorous conditions should be fulfilled at the same time: ion must occupy the tetrahedral position and have four d-electrons. Also, 


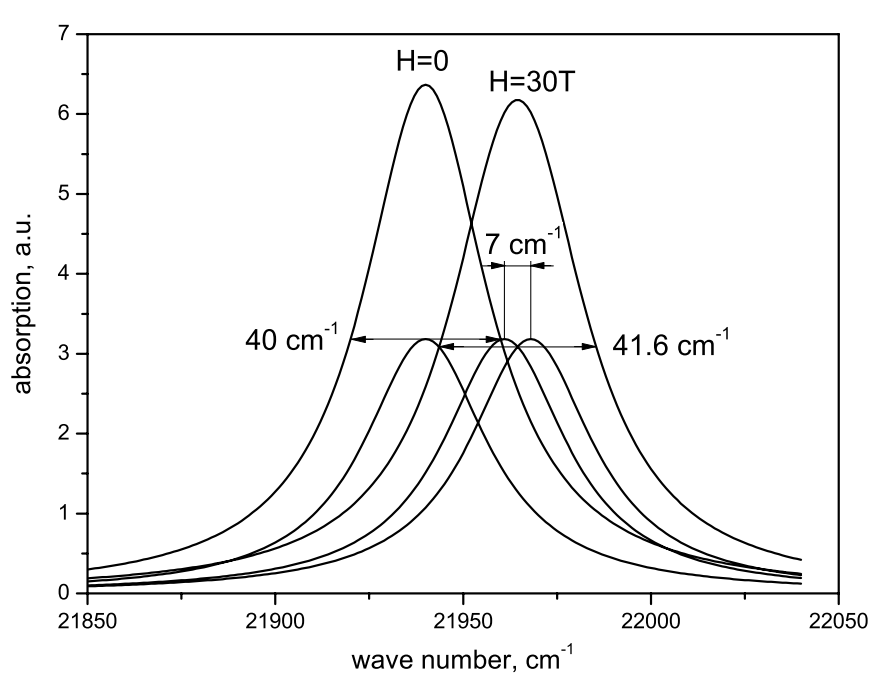

Fig. 8. Modelling shape of the absorption line.

preferable oxidation state of element is +4 . Amongst $3 \mathrm{~d}$ elements only iron satisfies these conditions. One more candidate is the $4 \mathrm{~d}$ element ruthenium $[10,11]$.

Observed absorption line could not be ascribed to the $\mathrm{Fe}^{+3}$ ion at tetrahedral position, because in this case the upper level should have $S=3 / 2$ and transitions between states with different spins are forbidden. Thus we rule out +3 as oxidation state of iron. The main argument that contradicts this conclusion is observation of EPR in Fe-doped sillenites [12,13], whereas $\mathrm{Fe}^{4+}$ is non-Kramers ion and which is usually difficult to detect by EPR. But EPR is a very sensitive method and a signal from $\mathrm{Fe}^{+3}$ ions was observed in a nominally pure $\mathrm{Bi}_{12} \mathrm{SiO}_{20}$ representing a concentration of approximately $10^{16}-10^{17}$ impurity ions per $\mathrm{cm}^{3}$ [12], that is $20-200$ times smaller than the doping level of our samples. Our experiments could not exclude existence of $\mathrm{Fe}^{+3}$ with such low content. On the other hand, there are example of large EPR signals from non-Kramers ions $\mathrm{Fe}^{2+}$ and $\mathrm{Fe}^{4+}$ [14]. In our case, transitions $2 \rightarrow 3$ and $7 \rightarrow 8$ (Fig. 7) are good candidates for EPR. Thus two EPR lines with $g=2$ and $g=1.5$ should be observed.

Change of transparency (Fig. 6) is a clear demonstration of the photochromic and photorefractive effects, which arise due to photo-excitation of electrons from impurity levels located in energy gap of insulator to the conduction band and subsequent capturing by shallow traps $[15,16]$. Obvious correlation between the background absorption and intensity of $\mathrm{Fe}^{4+}$ absorption line seen from Figure 6 indicates that iron dopant is responsible for the photochromic and photorefractive effects.

Obtained results may be interpreted with an energyband diagram (Fig. 9). According to [17] the band gap of $\mathrm{Bi}_{12} \mathrm{SiO}_{20}$ is equal to $3.4 \mathrm{eV}$ at temperatures below $80 \mathrm{~K}$. Ion $\mathrm{Fe}^{4+}$ has four spare $3 \mathrm{~d}$ electrons, compared with $\mathrm{Si}^{4+}$. When $\mathrm{Fe}^{4+}$ substitutes $\mathrm{Si}^{4+}$, 3d level of these electrons $(D)$ should be adjusted to the Fermi level which coincides with the top of valence band. But tetrahedral environment splits level $D$ into two levels - the lower orbital singlet $(E)$ and upper orbital triplet $T_{2}$ [9], each containing

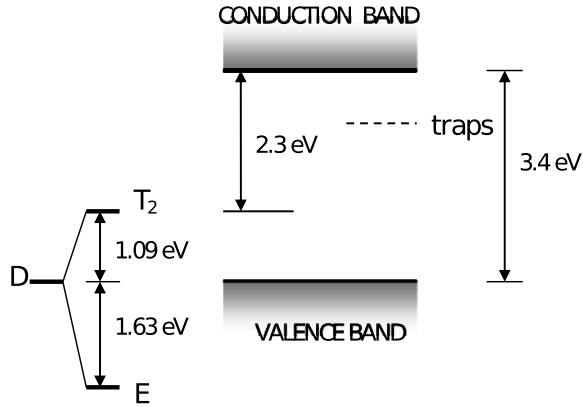

Fig. 9. Energy-band diagram of Fe-doped $\mathrm{Bi}_{12} \mathrm{SiO}_{20}$.

two electrons. Total splitting $\Delta=T_{2}-E=2.72 \mathrm{eV}$ was calculated from position of the observed absorption line at $21950 \mathrm{~cm}^{-1}, T_{2}-D=2 / 5 \Delta=1.09 \mathrm{eV}, D-E=3 / 5 \Delta=$ $1.63 \mathrm{eV}$. Thus level $T_{2}$ gets in the energy gap, making a dopant level essential for the photochromic and photorefractive effects. Distance from this dopant level to the bottom of conduction band is about $2.3 \mathrm{eV}$, that is smaller than the energy of blue light $(2.64 \mathrm{eV})$ and larger than the energy of red light $(1.94 \mathrm{eV})$. Distance from shallow traps level to the bottom of conduction band is much smaller because even infrared illumination can restore population of dopant level [18]. This energy band diagram explains influence of blue and red light illumination on the magnetoelectric effect in Fe-doped $\mathrm{Bi}_{12} \mathrm{SiO}_{20}$ single crystal.

White illumination used in observation of the Zeeman effect was obtained with a LED. According to the datasheet, its spectrum consists of two wide bands centred in blue and red regions. As it follows from the magnetoelectric effect investigations, blue light diminishes the number of $\mathrm{Fe}^{4+}$ ions and increases the number of $\mathrm{Fe}^{5+}$ ions, whereas red light restores the number of $\mathrm{Fe}^{4+}$ ions. This explains why amplitude of absorption line does not depend on duration of optical experiment performed with white LED illumination.

\section{Conclusions}

In this work we confirm the previous work results [4], the explanation of the influence of illumination on the magnetoelectric effect in sillenites. One experimental result was not mentioned in [4]. We have measured the piezoelectric effect in Cr-doped $\mathrm{Bi}_{12} \mathrm{TiO}_{20}$ at $T=4.2 \mathrm{~K}$ before and after half an hour illumination with the red light. No change in the piezoelectric coefficient was observed, whereas the same illumination drastically changes the magnetoelectric effect. This result excludes an extrinsic [19], i.e. caused by the magnetostriction, origin the magnetoelectric effect in sillenites. $^{2}$

The magnetic moment and the magnetoelectric effect in Fe-doped $\mathrm{Bi}_{12} \mathrm{SiO}_{20}$ were measured in high magnetic field. Obtained results are consistent with the +4 oxidation state of iron dopant. Illumination with the blue light changes sign of the magnetoelectric effect. Change in sign

\footnotetext{
${ }^{2}$ Contribution of the magnetostriction to the magnetoelectric effect in the paramagnetic piezoelectric $\mathrm{NiSO}_{4} \cdot 6 \mathrm{H}_{2} \mathrm{O}$ was separated in $[20]$.
} 
of the magnetoelectric effect was ascribed to the change of oxidation state of iron dopant from +4 to +5 . An absorption line near the absorption edge was observed at $T=4.2 \mathrm{~K}$. This line is possible only for ion $\mathrm{Fe}^{+4}$ at tetrahedral position. Measurements of the Zeeman effect fully confirm this conclusion. Thus all obtained results point to the +4 oxidation state of iron dopant in $\mathrm{Bi}_{12} \mathrm{SiO}_{20}$ that was not even considered in any preceding investigation.

We would like to thank Dr. M. Krasin'kova for providing us with Fe-doped $\mathrm{Bi}_{12} \mathrm{SiO}_{20}$ single crystals and G. Gajda for an assistance in measurements using long-pulse magnet.

\section{Author contribution statement}

K. Filar - cutting and polishing samples, performing experiment, discussion results and preparing paper. V.I. Nizhankovskii - idea, calculations, discussion results and preparing paper.

Open Access This is an open access article distributed under the terms of the Creative Commons Attribution License (http://creativecommons.org/licenses/by/4.0), which permits unrestricted use, distribution, and reproduction in any medium, provided the original work is properly cited.

\section{References}

1. O. Sato, T. Iyoda, A. Fujishima, K. Hashimoto, Science 49, 271 (1996)

2. W. Wardzyński, H. Szymczak, K. Pataj, T. Łukasiewicz, J. Zmija, J. Phys. Chem. Solids 41, 767 (1982)
3. M.G. Jeni, L.E. Halliburton, J. Appl. Phys. 64, 2022 (1988)

4. K.P. Filar, G.P. Gajda, V.I. Nizhankovskii, Phys. Status Solidi B 253, 473 (2016)

5. S.C. Abrahams, P.B. Jamieson, J.L. Bernstein, J. Chem. Phys. 47, 4034 (1967)

6. E. Suzuki, N. Iyi, K. Kitamura, J. Korean Phys. Soc. 32, S173 (1998)

7. P. Spitzer, C. Zierhofer, E. Hochmair, BioMed. Eng. OnLine 5, 13 (2006)

8. G.A. SlacK, F.S. Ham, R.M. Chrenko, Phys. Rev. 152, 376 (1966)

9. A. Abragam, B. Bleany, Paramagnetic resonance of transition ions (Clarendon Press, Oxford, 1970)

10. V. Marinova, S.H. Lin, V. Sainov, M. Gospodinov, K.Y. Hsu, J. Opt. A: Pure Appl. Opt. 5, S500 (2003)

11. F. Ramaz, L. Rakitina, M. Gospodinov, B. Briat, Opt. Mater. 27, 15471559 (2005)

12. W. Wardzyński, M. Baran, H. Szymczak, Physica 111B, $47(1981)$

13. H.J. von Bardeleben, J. Phys. D: Appl. Phys. 16, 29 (1983)

14. U. Kaufmann, Phys. Rev. B 14, 1848 (1976)

15. T.J. Hall, R. Jaura, L.M. Connors, P.D. Foote, Prog. Quantum Electron. 10, 77 (1985)

16. B.I. Sturman, V.M. Fridkin, Photovoltaic and photorefractive effects in noncentrosymmetric materials (Gordon and Breach Science Publishers, Amsterdam, 1992)

17. S.L. Hou, R.B. Lauer, R.E. Aldrich, J. Appl. Phys. 44, $2652(1973)$

18. A.A. Kamshillin, M.P. Petrov, Sov. Phys. Solid State 23, $1811(1981)$

19. M. Fiebig, J. Phys. D: Appl. Phys. 38, R123 (2005)

20. A.I. Kharkovskiy, Y.V. Shaldin, V.I. Nizhankovskii, J. Exp. Theor. Phys. 117, 1071 (2013) 